\title{
Antifungal Effects against Phaeoisariopsis personata under Greenhouse Conditions and Phytochemical Analysis of Jatropha curcas Leaf Extracts
}

\author{
Magreth Francis $^{1,2^{*}}$, Musa Chacha ${ }^{1}$, Patrick A. Ndakidemi ${ }^{1}$ and Ernest R. Mbega ${ }^{1}$ \\ ${ }^{1}$ School of Life Sciences and Bio-engineering, The Nelson Mandela African Institution of Science and Technology (NM-AIST), \\ P.O Box 447, Arusha, Tanzania \\ ${ }^{2}$ Centre for Research, Agricultural Advancement, Teaching Excellence and Sustainability in Food and Nutrition Security \\ (CREATES- FNS), Arusha, Tanzania
}

*For correspondence: maggyfrancy7919@gmail.com; francism@nm-aist.ac.tz

Received 03 February 2021; Accepted 01 May 2021; Published 10 July 2021

\begin{abstract}
The study was conducted to test the antifungal efficacy of $J$. curcas leaf extracts against Phaeoisariopsis personata (causal pathogen for groundnut late leaf spot disease) under in vivo conditions, and to identify important phytochemical constituents exhibiting antifungal properties. The results showed that the greatest reduction of late leaf spot disease incidence was achieved by all the Jatropha curcas leaf extracts at the highest concentration $\left(0.5 \mathrm{mg} \mathrm{mL}^{-1}\right)$ as $36.89,36.59$ and $24.67 \%$ for chloroform, ethyl acetate and methanolic extracts, respectively. Subsequently, J. curcas leaf extracts treatments enhanced the growth and yield of groundnut compared with the control (untreated). The antifungal effects of $J$. curcas were supported by the presence of phytochemical constituents identified by GC-MS. Hexadecane; $n$-hexadecanoic acid; phenol, 2 , 4 bis (-dimethylethyl); phytol and hexadecanoic methyl ester were detected as major phytocompounds in $J$. curcas leaf extracts that were possibly responsible for the antifungal activity. (C) 2021 Friends Science Publishers
\end{abstract}

Keywords: Biological control; Efficacy; Groundnut; Late leaf spot; Phytocompounds

\section{Introduction}

Groundnut (Arachis hypogaea L.) is a vital oil kernel crop in the tropics and subtropics countries (Pasupuleti et al. 2013). The groundnut is cultivated in many countries between latitudes $40^{\circ} \mathrm{N}$ and $40^{\circ} \mathrm{S}$ in semiarid tropics and subtropics (Kayondo et al. 2014). Groundnut seeds contain about $12-15 \%$ carbohydrates, $25-30 \%$ protein and $40-50 \%$ fats (Saeed and Hassan 2009). Additionally, groundnuts contain a good source of vitamin, dietary fibres, and minerals such as niacin, magnesium, iron, phosphorus, calcium, zinc, and riboflavin (Tshilenge-Lukanda et al. 2013). In addition, groundnut as a legume crop improves soil fertility by converting the atmospheric nitrogen to nitrates, ammonia, and organic nitrogen (Pasupuleti et al. 2013). Thus, groundnut production plays a great role in developing and developed nations in improving the economic status (Tsigbey et al. 2003).

In Tanzania, the crop is cultivated mainly by smallholder farmers in Tabora, Mtwara, Dodoma, Singida, Shinyanga and Mwanza regions. The crop acts as a good source of food, cash crop, and animal feed (Osei et al. 2013). Despite the importance of groundnut in Tanzania, its average yield is still low amounting to 960 $\mathrm{kg} \mathrm{ha}^{-1}$ as opposed to the predictable yield potential of $2500 \mathrm{~kg} \mathrm{ha}^{-1}$ in developing countries (Philipo and Nchimbi-Msolla 2019). The crop is mainly constrained by drought stress, low level of inputs, foliar fungal diseases, and insect pest attacks.

The foliar fungal diseases namely, early leaf spot, late leaf spot, and rust are among the most destructive diseases, which account for huge economic yield losses (Naidu et al. 1999). The late leaf spot disease is one among the three diseases, which has been identified as a major constrain wherever groundnut is cultivated in Tanzania. The late leaf spot disease infection reduces the photosynthetic area by causing intense lesions on stems, leaves, and petioles consequently leading to defoliation and hence high yield losses (Monfort et al. 2004; Khedikar et al. 2010). According to Ghewande (1989), when the late leaf spot disease attacks groundnuts, it reduces about 80 and $93 \%$ canopy carbon exchange rate and carbon uptake respectively. Late leaf spot disease also causes adverse outcomes on seed and folder quality becoming unsuitable for animal feed (Monfort et al. 2004).

Efforts have been made in controlling late leaf spot disease through the development and the use of synthetic

To cite this paper: Francis M, M Chacha, PA Ndakidemi, ER Mbega (2021). Antifungal effects against Phaesariopsis personata under greenhouse conditions and phytochemical analysis of Jatropha curcas leaf extracts. Intl J Agric Biol 26:231-240 
fungicides, which have been proven effective. However, the effectiveness of synthetic fungicides depends upon multiple fungicide applications; hence, smallholder farmers cannot afford the cost. Moreover, there are issues of environmental and health concerns (Jordan et al. 2012). The plants possess phytochemical compounds, which are effective against different pests including insects, fungi, virus, nematode, bacteria etc. (Khan et al. 2020; Javaid et al. 2020, 2021). Thus, plant based materials can be used as a substitute for synthetic pesticides in order to ensure the safety of human and the environment (Engindeniz and Engindeniz 2013; Khan and Javaid 2020; Banaras et al. 2021).

The Jatropha curcas belonging to Euphorbiaceae family is a multipurpose plant that survives in both tropics and arid regions. Almost all the plant parts of Jatropha are reported to possess antimicrobial potential against disease causing pathogens including fungi (Prasad et al. 2012; Dada et al. 2014). Moreover, J. curcas leaf possesses important compounds such as sterols, terpenes, flavonoids, saponin and steroids steroids, which play a great antifungal role (Nwosu and Okafor 1995; Campa et al. 2008; Saetae and Worapot 2010). Many findings confirm the antifungal potential of $J$. curcas in managing fungal diseases. A study by Thangavelu et al. (2004) found that $J$. curcas was effective in managing banana anthracnose disease. In addition, $J$. curcas leaf extracts were found effective in managing Azolla disease caused by Sclerotium spp. (Garcia and Lawas 1990). However, little is known about the effectiveness of $J$. curcas against $P$. personata pathogen causing the late leaf spot disease on groundnuts. Thus, the present study evaluated the efficacy of $J$. curcas against the groundnut late leaf spot disease and analysed the possible compounds exhibiting antifungal properties.

\section{Materials and Methods}

\section{Isolation of pathogen and culture preparation}

Groundnut leaves showing symptoms of $P$. personata (circular spots underneath the leaflets) were obtained from the farmer's fields in Singida and Dodoma regions, Tanzania. Fungal isolation was done by adopting the method described by Kishore et al. (2007) with some modification. The diseased portions of leaves were cut into pieces $(0.5-1 \mathrm{~cm})$ and sterilized with $5 \% \mathrm{NaOCl}$. Thereafter, the pieces were rinsed three times in the sterilized distilled water and dried on a blotter paper in Petri dishes. Thereafter, the pieces were plated onto potato dextrose agar (PDA) medium in a laminar hood then incubated at $28 \pm 2^{\circ} \mathrm{C}$ for 7 days. After the emergence of mycelial growth, each fungal colony was sub-cultured into fresh PDA plates and incubated at $28 \pm 2^{\circ} \mathrm{C}$ for 7 days to obtain the pure culture of $P$. personata. The $P$. personata pathogen was identified by a single spore method under compound microscope (magnification 40X).

\section{Preparation of $\boldsymbol{J}$. curcas leaf extracts}

$J$. curcas leaves were collected from different parts in Arusha, Tanzania. The leaves were washed thoroughly and then air-dried at room temperature. Thereafter, these were pounded into powder. The fine powdered leaf sample $(1 \mathrm{~kg})$ was successively extracted through chloroform, ethyl acetate, and lastly methanol (48 $\mathrm{h}$ in each) at standard room temperature. Then, the leaf extracts were filtered with a Whatman No.1 filter paper and concentrated by a rotary evaporator. The product, which was a dark sticky semisolid extracts, was then stored under cold condition $\left(4^{\circ} \mathrm{C}\right)$ for further experiment.

\section{Greenhouse experiment on antifungal assay}

The study was carried out to test the effectiveness of methanol, chloroform and ethyl acetate leaf extracts of $J$. curcas against late leaf spot disease under greenhouse condition. Three groundnut seeds (Upendo variety) were grown in a plastic pots filled with a mixture of black soil (later thinned to one). The plants were artificially inoculated by $J$. curcas conidial suspension 30 days after sowing (DAS). After inoculation, the plants were shielded with plastic sheets for $48 \mathrm{~h}$ to maintain leaf wetness during the nights. Four foliar sprays were applied onto the plants each $J$. curcas leaf extract at $0.1,0.25$ and $0.5 \mathrm{mg} \mathrm{mL}^{-1}$ concentration, chlorothalonil $\left(2 \mathrm{~mL} \mathrm{~L}^{-1}\right)$ (positive control) and sterile distilled water (negative control) using a hand sprayer at 14 days interval. The plants were sprayed starting from 48 DAS and completed two weeks before the harvest. The experiment was inspected often and the data on disease, growth, and yield were recorded. The trial was set in a completely randomized design replicated three times. The experiment was repeated twice. The disease incidence was assessed on each plant by evaluating the percent of the infected leaves per plant by adopting the formula by Subrahmanyam et al. (1995).

Disease incidence $(\%)=\frac{\text { Number of infected leaves per plant }}{\text { Totalnumber of leaves per plant }} \times 100$

The late leaf spot disease severity under different treatments was scored using (1-9) disease rating scale (Chiteka et al. 1988).

\section{Gas chromatography mass spectroscopy analysis}

The phytochemical analysis of $J$. curcas leaf extracts was done using gas chromatography-mass spectroscopy (GCMS) on Agilent technologies 7890A GC connected to Agilent 5975 MSD (Agilent technology, USA), comprising a $30 \mathrm{~m}$ length and film $0.25 \mu \mathrm{m}$ and internal diameter of $0.250 \mathrm{~mm}$ and temperature limit of $50^{\circ} \mathrm{C}$ to $340^{\circ} \mathrm{C}\left(360^{\circ} \mathrm{C}\right)$. The inert gas helium was used as a carrier gas with $1.2 \mathrm{~mL}$ $\min ^{-1}$ flow rate. The inlet temperature was $250^{\circ} \mathrm{C}$ and the total running time was $35 \mathrm{~min}$. The obtained peaks were 
compared with the known compounds spectra stored in the National Institute Standard and Technology library.

\section{Data analysis}

Data were subjected to 3-way ANOVA (analysis of variance) in factorial arrangement, using STATISTICA program. The treatment means were compared by applying Fischer's least significant difference (LSD) at 5\% level of significance.

\section{Results}

In this study, the effectiveness of $J$. curcas leaf extracts against the late leaf spot disease was determined by observing their effect on reducing the disease incidence and severity. Moreover, growth and yield attributes were also assessed. Three leaf extract of J. curcas (methanolic, chloroform, and ethyl acetate), one standard fungicide chlorothalonil (positive control) and one negative control (distilled water) as foliar spray were used as treatments against late leaf spot disease. The effects of treatments, solvents and concentrations on late leaf spot disease incidence, severity, growth and yield attributes are presented in Table 1, 2 and 3.

The late leaf spot disease varied significantly $(P \leq$ 0.001 ) with the effect to treatments. The plants treated by Jatropha leaf extracts had lower late leaf spot disease incidence $(13.33 \%)$ similar to the standard fungicide chlorothalonil $(5.41 \%)$. Moreover, the late leaf spot disease incidence differed significantly $(P \leq 0.01)$ with the type of solvents used for extraction. Methanolic leaf extract of $J$. curcas had lower late leaf spot disease incidence $(24.7 \%)$ as compared to chloroform $(36.89 \%)$ and ethyl acetate $(36.59 \%$,) extracts (Table 1). Moreover, the results showed that the late leaf spot disease incidence and severity differed significantly $(P \leq 0.001)$ from the effect of $J$. curcas leaf extracts concentration. $J$. curcas leaf extracts at the highest concentration $\left(0.5 \mathrm{mg} \mathrm{mL}^{-1}\right)$ significantly reduced the late leaf spot disease incidence as compared to the lowest concentration $\left(0.1 \mathrm{mg} \mathrm{mL}^{-1}\right)$ (Table 1$)$.

Growth parameters varied significantly $(P \leq 0.001)$ with the effect of treatments, solvents and concentrations of $J$. curcas leaf extracts (Table 2). The plants treated with chlorothalonil and $J$. curcas had taller shoots and big number of leaves per plant both at flowering and maturity $(38.59 \mathrm{~cm}, 8.93)(47.26 \mathrm{~cm}, 12.89),(27.78 \mathrm{~cm}, 6.74)$ and $(36.07 \mathrm{~cm}, 9.48)$ respectively compared with the control. Similarly, growth parameter differed significantly $(P \leq 0.05)$ from the type of solvents used for extraction. The methanolic and chloroform leaf extracts of J. curcas had taller shoots $(30.48 \mathrm{~cm}, 29.56 \mathrm{~cm}),(38.63 \mathrm{~cm}$, and 36.96 $\mathrm{cm}$ ) at both flowering and maturity respectively compared with ethyl acetate extracts $(27.52 \mathrm{~cm}, 34.93 \mathrm{~cm})$. Additionally, shoot length and big number of leaves per plant differed significantly $(P \leq 0.001)$ at different $J$. curcas leaf extracts concentrations. The plants treated with plant extracts at the highest concentration $\left(0.5 \mathrm{mg} \mathrm{mL}^{-1}\right)$ had taller shoots and bigger number of leaves per plant at flowering and maturity (31.89 cm, 7.88), (39.9 $3 \mathrm{~cm}, 9.93)$, respectively compared with the control $(25.91 \mathrm{~cm}, 5.82)$, $(33.48 \mathrm{~cm}, 7.81)$ at both flowering and maturity (Table 2$)$.

Likewise, yield attributes components varied significantly $(P \leq 0.001)$ with the treatments where $J$. curcas leaf extracts had bigger number of pods per plant, seeds per plant and seed yield (ton ha ${ }^{-1}$ ). J. curcas was less similar to the standard fungicides (chlorothalonil) that is, the number of pods per plant (32.9), the number of seeds per plant (61.2) and seed yield (1.6 ton ha ${ }^{-1}$ ) (Table 3). Yield data did not differ significantly with the effect to solvents, similar results were observed. The yield attributes differed significantly $(P \leq 0.01)$ with the effect to $J$. curcas leaf extracts concentration. $J$. curcas leaf extracts at the highest concentration had bigger number of pods plant ${ }^{-1}$ (34.59), the number of seeds per plant (63.19), 100 seed weight (53.09 g) and seed yield (1.85 ton ha ${ }^{-1}$ ) (Table 3 ).

The GC-MS results led to the identification of different phytochemical constituents from fractions of ethyl acetate, methanolic, and chloroform leaf extracts of $J$. curcas. The mass spectra of the detected compounds from methanolic, chloroform and ethyl acetate leaf extracts of $J$. curcas were compared with the spectra of the recognized compounds in the NIST library. The name of compound, molecular weight, retention time, and molecular formula of the compounds contained in these leaf extracts are presented in Tables 4, 5 and 6. The following phytoconstituents with antifungal properties were recognized by GC-MS from the chloroform leaf extract of $J$. curcas; dodecane; 2,6,11trimethyl-2-tetradecene; tetradecane; pentadecane; octacosane; sulfurous acid butyl decyl ester; 2-bromo heneicosane; phenol 2,4-bis (1, 1-dimethylethyl); hexadecane; heptadecane; heptacosane; 2,4dimethyldodecane; n-hexadecanoic acid; ethanol 2(octadecyloxy)-; hentriacontane; geranylgeraniol; octadecane; 12-methyl-E-E-2 13-octadecadien-1-ol; tetradecanal and cyclotetracosane (Table 4). Among them $n$ hexadecanoic acid (7.89\%); phenol 2,4-bis (1,1dimethylethyl) (4.04\%); cyclotetracosane (1.23\%); hexadecane $(1.20 \%)$ and octacosane $(1.02 \%)$ were the major identified phytoconstituents compounds (Table 4).

From ethyl acetate leaf extract of $J$. curcas, the following phytoconstituents with antifungal activity were identified by GC-MS; 1,2,3-ropanetriol; monoacetate; 2,5pyrrolidinedione; thiomorpholine; methyl salicylate; triacetin, 1-naphthalenol; 8-hexadecenal; 14-methyl-, (Z)-, undecane; phenol, 2,4-bis (1,1-dimethylethyl); hexadecane; heptadecane; $1 \mathrm{H}$-indene 1-ethylideneoctahydro-7 a-methylcis-, E-14-hexadecenal; 1-tetradecene; tetramethyl-2hexadecen-1-ol; 9,12-octadecadienoic acid (Z,Z); 5eicosene, $(\mathrm{E})$; hexadecanoic acid ethyl ester; 2-methyl-Z,Z3,13-octadecadienol,; (Z)-; $n$-hexadecanoic acid; phytol; 9,12,15-octadecatrienoic acid ethyl ester; (Z,Z,Z)-; 
Francis et al. / Intl J Agric Biol, Vol 26, No 2, 2021

Table 1: Late leaf spot disease incidence and severity as affected with treatments, solvent and concentration

\begin{tabular}{|c|c|c|}
\hline Factors & Incidence & Severity \\
\hline \multicolumn{3}{|l|}{ Treatments } \\
\hline Jatropha curcas & $13.33 \pm 2.02^{\mathrm{b}}$ & $2.26 \pm 0.31^{\mathrm{b}}$ \\
\hline Chlorothalonil & $5.41 \pm 1.07^{\mathrm{a}}$ & $1.33 \pm 0.22^{\mathrm{a}}$ \\
\hline Control & $89.41 \pm 0.92^{\mathrm{c}}$ & $8.96 \pm 0.04^{\mathrm{c}}$ \\
\hline \multicolumn{3}{|l|}{ Solvents } \\
\hline Chloroform & $36.89 \pm 7.49^{\mathrm{b}}$ & $3.89 \pm 0.63^{\mathrm{b}}$ \\
\hline Ethyl acetate & $36.59 \pm 7.59^{\mathrm{b}}$ & $3.00 \pm 0.59^{\mathrm{b}}$ \\
\hline Methanol & $24.67 \pm 7.60^{\mathrm{a}}$ & $2.67 \pm 0.63^{\mathrm{a}}$ \\
\hline \multicolumn{3}{|l|}{ Concentrations } \\
\hline $0.1 \mathrm{mg} \mathrm{mL}^{-1}$ & $41.89 \pm 7.36^{\mathrm{c}}$ & $5.42 \pm 0.53^{\mathrm{c}}$ \\
\hline $0.25 \mathrm{mg} \mathrm{mL}^{-1}$ & $35.93 \pm 7.39^{\mathrm{b}}$ & $4.00 \pm 0.59^{\mathrm{b}}$ \\
\hline $0.5 \mathrm{mg} \mathrm{mL}^{-1}$ & $20.33 \pm 7.76^{\mathrm{a}}$ & $2.07 \pm 0.69^{\mathrm{a}}$ \\
\hline \multicolumn{3}{|l|}{ 3-WAY ANOVA (F-value) } \\
\hline Treatments & $4332.52 * * *$ & $512.564 * * *$ \\
\hline Solvents & $2390.38 * *$ & $1.14 * *$ \\
\hline Concentrations & $1.62 * * *$ & $20.34 * * *$ \\
\hline Treatments*Solvents & $37.11 \mathrm{~ns}$ & $4.555^{* *}$ \\
\hline Treatments*Concentrations & $1.57 \mathrm{~ns}$ & $8.04 * * *$ \\
\hline Solvents*Concentrations & $7.751 * * *$ & $0.38 \mathrm{~ns}$ \\
\hline Treatments*Solvents*Concentrations & $0.381 \mathrm{~ns}$ & $0.56 \mathrm{~ns}$ \\
\hline
\end{tabular}

Table 2: Growth attributes of groundnut Upendo genotype as affected by treatments, solvents and concentrations

\begin{tabular}{|c|c|c|c|c|}
\hline Factors & $\begin{array}{l}\text { Shoot length }(\mathrm{cm}) \\
\text { flowering }\end{array}$ & $\begin{array}{l}\text { Number of branches at } \\
\text { flowering }\end{array}$ & $\begin{array}{l}\text { Shoot length at } \\
\text { maturity }\end{array}$ & $\begin{array}{l}\text { Number of branches at } \\
\text { maturity }\end{array}$ \\
\hline \multicolumn{5}{|l|}{ Treatments } \\
\hline Jatropha curcas & $27.78 \pm 1.16^{\mathrm{b}}$ & $6.74 \pm 0.27^{\mathrm{b}}$ & $36.07 \pm 1.22^{\mathrm{b}}$ & $9.48 \pm 0.43^{\mathrm{b}}$ \\
\hline Chlorothalonil & $38.59 \pm 1.10^{\mathrm{c}}$ & $8.93 \pm 0.35^{\mathrm{c}}$ & $47.26 \pm 1.19^{c}$ & $12.89 \pm 0.47^{\mathrm{c}}$ \\
\hline Control & $21.19 \pm 0.72^{\mathrm{a}}$ & $4.85 \pm 0.17^{\mathrm{a}}$ & $27.19 \pm 0.71^{\mathrm{a}}$ & $4.48 \pm 0.14^{\mathrm{a}}$ \\
\hline \multicolumn{5}{|l|}{ Solvents } \\
\hline Chloroform & $29.56 \pm 1.76^{\mathrm{b}}$ & $6.96 \pm 0.42^{\mathrm{a}}$ & $36.96 \pm 2.00^{\mathrm{b}}$ & $9.33 \pm 0.85^{\mathrm{b}}$ \\
\hline Ethyl acetate & $27.52 \pm 1.63^{\mathrm{a}}$ & $6.59 \pm 0.40^{\mathrm{a}}$ & $34.93 \pm 1.79^{\mathrm{a}}$ & $8.15 \pm 0.71^{\mathrm{a}}$ \\
\hline Methanol & $30.48 \pm 1.75^{\mathrm{b}}$ & $6.69 \pm 0.45^{\mathrm{a}}$ & $38.63 \pm 1.93^{c}$ & $9.37 \pm 0.73^{\mathrm{b}}$ \\
\hline \multicolumn{5}{|l|}{ Concentrations } \\
\hline $0.1 \mathrm{mg} \mathrm{mL}^{-1}$ & $25.91 \pm 0.95^{\mathrm{a}}$ & $5.82 \pm 0.31^{\mathrm{a}}$ & $33.48 \pm 1.53 \mathrm{a}$ & $7.81 \pm 0.62^{\mathrm{a}}$ \\
\hline $0.25 \mathrm{mg} \mathrm{mL}^{-1}$ & $29.58 \pm 1.10^{\mathrm{b}}$ & $6.81 \pm 0.39^{b}$ & $37.11 \pm 1.95^{\mathrm{b}}$ & $9.11 \pm 0.72^{b}$ \\
\hline $0.5 \mathrm{mg} \mathrm{mL}^{-1}$ & $31.89 \pm 1.18^{\mathrm{c}}$ & $7.88 \pm 0.47^{\mathrm{c}}$ & $39.93 \pm 2.07^{\mathrm{c}}$ & $9.93 \pm 0.89^{c}$ \\
\hline \multicolumn{5}{|l|}{ 3-Way ANOVA (F-value) } \\
\hline Treatments & $153.15^{* * *}$ & $108.23 * * *$ & $179.32 * * *$ & $252.32 * * *$ \\
\hline Solvents & $4.56^{*}$ & $1.190 \mathrm{~ns}$ & $6.098 * *$ & $6.819 * *$ \\
\hline Concentrations & $17.54 * * *$ & $28.012 * * *$ & $18.49 * * *$ & $15.994 * * *$ \\
\hline Treatments*Solvents & $10.29 * * *$ & $4.226^{* *}$ & $8.58 * * *$ & $7.72 * * *$ \\
\hline Treatments*Concentrations & $2.251 \mathrm{~ns}$ & $3.51 *$ & $3.15^{*}$ & $4.74 * *$ \\
\hline Solvents*Concentrations & $0.15 \mathrm{~ns}$ & $1.048 \mathrm{~ns}$ & $0.09 \mathrm{~ns}$ & $0.18 \mathrm{~ns}$ \\
\hline Treatments*Solvents*Concentrations & $1.20 \mathrm{~ns}$ & $0.896 \mathrm{~ns}$ & $0.923 \mathrm{~ns}$ & $0.96 \mathrm{~ns}$ \\
\hline
\end{tabular}

heptadecanoic acid ethyl ester and eicosane (Table 5). The major phytoconstituents were phytol (9.31\%); thiomorpholine (4.83\%); hexadecanoic acid ethyl ester (3.97\%); phenol 2,4-bis (1,1-dimethylethyl) (3.37\%); 9,12,15-octadecatrienoic acid ethyl ester, (Z,Z,Z)- (2.75\%); 5-eicosene, (E)- (2. 11\%) and 1-heneicosyl (1.92\%) (Table $5)$.

The phytoconstituents with antifungal property identified by GC-MS in J. curcas methanolic leaf extract were; 1,2,3-propanetriol monoacetate; methyl salicylate; 2undecanone; decanoic acid methyl ester; 2-methoxy-4vinylphenol; tert-hexadecanethiol; phenol 2,6-dimethoxy; tetradecane; cyclotetradecane; pentanoic acid ethyl ester; 2propenoic acid 3-phenyl- methyl ester; diphenyl ether; pentadecane; tridecane; hexadecane; heptadecane; 17 pentatriacontene, 1-nonadecene; E-15-heptadecenal; 8hexadecenal 14-methyl; cyclopentadecane; hexadecanoic acid methyl ester; 1-octadecene; 2-methyl-Z, Z-3, 13octadecadienol; oleic acid, 9,17-octadecadienal, (Z); 2methyl-Z,Z-3,13-octadecadienol; 9, 12-octadecadienoic acid (Z,Z)-methyl ester; phytol; octadecanoic acid methyl ester; behenic alcohol; octadecanoic acid ethyl ester; 3,7,11,15tetramethyl-2-hexadecen-1-ol; 9,17-octadecadienal; eicosane and docosanoic acid methyl ester (Table 6). Phytol (26.75\%); hexadecanoic acid methyl ester (14.32\%); octadecanoic acid methyl ester $(2.79 \%)$ and 9,12octadecadienoic acid (Z,Z)- methyl ester (2.33\%) were identified as major phytoconstituents (Table 6). 
Management of Groundnut Late Leaf Spot Disease / Intl J Agric Biol, Vol 26, No 2, 2021

Table 3: Yield attributes of groundnut Upendo genotype as affected by treatments, solvents and concentrations

\begin{tabular}{|c|c|c|c|c|}
\hline Factors & Number of pods/plant & Number of seeds/plant & 100 kernel weight $(\mathrm{g})$ & Seed yield (tonnes/ha) \\
\hline \multicolumn{5}{|l|}{ Treatments } \\
\hline Jatropha curcas & $32.96 \pm 1.13^{\mathrm{b}}$ & $61.15 \pm 2.25^{\mathrm{b}}$ & $49.00 \pm 2.30^{\mathrm{b}}$ & $1.59 \pm 0.09^{\mathrm{b}}$ \\
\hline Chlorothalonil & $40.30 \pm 1.27^{\mathrm{c}}$ & $75.81 \pm 2.49^{\mathrm{c}}$ & $54.18 \pm 1.50^{\mathrm{c}}$ & $2.49 \pm 0.10^{\mathrm{c}}$ \\
\hline Control & $16.0 \pm 0.95^{\mathrm{a}}$ & $24.41 \pm 1.67^{\mathrm{a}}$ & $33.85 \pm 1.75^{\mathrm{a}}$ & $0.49 \pm 0.03^{\mathrm{a}}$ \\
\hline \multicolumn{5}{|l|}{ Solvents } \\
\hline Chloroform & $30.07 \pm 2.40^{\mathrm{b}}$ & $53.96 \pm 5.04^{\mathrm{a}}$ & $50.07 \pm 1.79^{\mathrm{a}}$ & $1.54 \pm 0.19^{\mathrm{a}}$ \\
\hline Ethyl acetate & $28.59 \pm 2.18^{\mathrm{a}}$ & $51.33 \pm 4.56^{\mathrm{a}}$ & $50.82 \pm 1.75^{\mathrm{a}}$ & $1.50 \pm 0.18^{\mathrm{a}}$ \\
\hline Methanol & $30.59 \pm 2.25^{\mathrm{b}}$ & $56.07 \pm 4.62^{\mathrm{a}}$ & $51.13 \pm 1.57^{\mathrm{b}}$ & $1.54 \pm 0.17^{\mathrm{a}}$ \\
\hline \multicolumn{5}{|l|}{ Concentration } \\
\hline $0.1 \mathrm{mg} \mathrm{mL}^{-1}$ & $24.59 \pm 2.00^{\mathrm{a}}$ & $43.85 \pm 4.15^{\mathrm{a}}$ & $49.16 \pm 1.52^{\mathrm{a}}$ & $1.20 \pm 0.14^{\mathrm{a}}$ \\
\hline $0.25 \mathrm{mg} \mathrm{mL}^{-1}$ & $30.07 \pm 2.18^{\mathrm{b}}$ & $54.33 \pm 4.55^{\mathrm{b}}$ & $49.78 \pm 1.71^{\mathrm{a}}$ & $1.52 \pm 0.17^{\mathrm{b}}$ \\
\hline $0.5 \mathrm{mg} \mathrm{mL}^{-1}$ & $34.59 \pm 2.23^{\mathrm{c}}$ & $63.19 \pm 4.76^{\mathrm{c}}$ & $53.09 \pm 1.82^{\mathrm{b}}$ & $1.85 \pm 0.20^{\mathrm{c}}$ \\
\hline \multicolumn{5}{|l|}{ 3-Way ANOVA (F-value) } \\
\hline Treatments & $282.57 * * *$ & $353.15^{* * * *}$ & $38.74 * * *$ & $248.92^{* * *}$ \\
\hline Solvents & $1.96 \mathrm{~ns}$ & $2.84 \mathrm{~ns}$ & $0.135^{*}$ & $0.15 \mathrm{~ns}$ \\
\hline Concentrations & $45.63 * *$ & $47.17 * *$ & $0.47 *$ & $25.883^{* * *} *$ \\
\hline Treatments*Solvents & $1.707 \mathrm{~ns}$ & $1.92 \mathrm{~ns}$ & $0.44 \mathrm{~ns}$ & $0.63 \mathrm{~ns}$ \\
\hline Treatments*Concentrations & $1.179 \mathrm{~ns}$ & $1.47 \mathrm{~ns}$ & $0.43 \mathrm{~ns}$ & $3.136^{*}$ \\
\hline Solvents*Concentrations & $4.033^{*}$ & $3.669 *$ & $0.45 \mathrm{~ns}$ & $1.774 \mathrm{~ns}$ \\
\hline Treatments*Solvents*Concentrations & $0.238 \mathrm{~ns}$ & $0.212 \mathrm{~ns}$ & $1.20 \mathrm{~ns}$ & $1.482 \mathrm{~ns}$ \\
\hline
\end{tabular}

Table 4: Phytochemical compounds with antifungal activity obtained from chloroform leaf extract of J. curcas

\begin{tabular}{|c|c|c|c|c|}
\hline Retention time (min) & Compound name & Molecular formula & Molecular weight (g/mol) & References \\
\hline 10.629 & Dodecane, 2,6,11-trimethyl- & $\mathrm{C}_{15} \mathrm{H}_{32}$ & 212.41 & (Zhang et al. 2015) \\
\hline 11.745 & 2-Tetradecene & $\mathrm{C}_{14} \mathrm{H}_{28}$ & 196.37 & (Shirani et al. 2017) \\
\hline 11.905 & Tetradecane & $\mathrm{C}_{14} \mathrm{H}_{30}$ & 198.39 & (Begum et al.2016) \\
\hline 12.958 & Octacosane & $\mathrm{C}_{28} \mathrm{H}_{58}$ & 394.76 & (Zhang et al.2018) \\
\hline 13.192 & Sulfurous acid butyl decyl ester & $\mathrm{C}_{16} \mathrm{H}_{34} \mathrm{O}_{3} \mathrm{~S}$ & 306.50 & (Sharma et al. 2019) \\
\hline 13.267 & Heneicosane & $\mathrm{C}_{21} \mathrm{H}_{44}$ & 296.57 & (Ebrahimabadi et al. 2016) \\
\hline 13.461 & Phenol 2,4-bis(1, 1-dimethylethyl) & $\mathrm{C}_{14} \mathrm{H}_{22} \mathrm{O}$ & 206.32 & (Manikandan et al. 2017) \\
\hline 14.011 & 2-Bromo dodecane & $\mathrm{C}_{12} \mathrm{H}_{25} \mathrm{Br}$ & 249.23 & (Manikandan et al. 2017) \\
\hline 14.503 & Hexadecane & $\mathrm{C}_{16} \mathrm{H}_{34}$ & 226.44 & (Zhang et al. 2015) \\
\hline 15.041 & Heptadecane, 9-octyl- & $\mathrm{C}_{25} \mathrm{H}_{52}$ & 352.68 & (Musa et al.2015) \\
\hline 15.401 & Heptacosane & $\mathrm{C}_{27} \mathrm{H}_{56}$ & 380.73 & (Bouzabata et al.2018) \\
\hline 16.002 & 2,4-Dimethyldodecane & $\mathrm{C}_{14} \mathrm{H}_{30}$ & 198.38 & (Begum et al. 2016) \\
\hline 16.488 & Pentadecane & $\mathrm{C}_{15} \mathrm{H}_{32}$ & 212.41 & (Yuan et al.2012; Zhang et al. 2015) \\
\hline 17.009 & Ethanol, 2-(octadecyloxy)- & $\mathrm{C}_{20} \mathrm{H}_{42} \mathrm{O}_{2}$ & 314.50 & (Mohy and Mohyeldin 2018) \\
\hline 18.067 & Octacosane & $\mathrm{C}_{28} \mathrm{H}_{58}$ & 394.76 & (Zhang et al.2018) \\
\hline 18.457 & Geranylgeraniol & $\mathrm{C}_{20} \mathrm{H}_{34} \mathrm{O}$ & 290.48 & (Ashraf et al. 2017) \\
\hline 18.542 & Octadecane & $\mathrm{C}_{18} \mathrm{H}_{38}$ & 254.49 & (Zhang et al. 2018) \\
\hline 18.869 & $n$-Hexadecanoic acid & $\mathrm{C}_{16} \mathrm{H}_{32} \mathrm{O}_{2}$ & 256.42 & (Omoruyi et al. 2014) \\
\hline 19.584 & 12-Methyl-E-E-2, 13-octadecadien-1-ol & $\mathrm{C}_{19} \mathrm{H}_{36} \mathrm{O}$ & 280.00 & (Vijayabaskar and Elango 2018). \\
\hline 20.013 & Tetradecanal & $\mathrm{C}_{14} \mathrm{H}_{28} \mathrm{O}$ & 212.37 & (Passos et al. 2003) \\
\hline 29.037 & Cyclotetracosane & $\mathrm{C}_{24} \mathrm{H}_{48}$ & 336.64 & (Bughio et al. 2017) \\
\hline
\end{tabular}

\section{Discussion}

The in vivo studies confirmed the efficacy of J. curcas by lowering the disease incidence and severity as the concentration increased. The lowest late leaf spot disease incidence and severity were achieved with both $J$. curcas leaf extracts, similar to the standard fungicide (chlorothalonil). This corresponds with the findings of Thangavelu et al. (2004), who revealed that the leaf extract of $J$. curcas effectively controlled Colletotrichum musae and Sclerotium spp. causal agents for anthracnose disease in banana and Azolla, respectively. Methanolic extracts showed the lowest late leaf spot disease incidence and severity compared to ethyl acetate and chloroform extracts. This suggests that more polar compounds extracted by methanol had antifungal property slightly greater than had those extracted by ethyl acetate and chloroform. This finding is consistent with the findings by Igbinosa et al. (2009) who revealed that, the stem bark methanolic extract of $J$. curcas inhibited the growth of Escherichia coli, Bacillus subtilis and Proteus vulgaris. Moreover, according to Kalimuthu et al. (2010) the methanolic extract of $J$. curcas inhibited Pseudomonas, Klebsiella, E. coli and Staphylococcus aureus. Moreover, the $J$. curcas leaf extracts at the highest concentration significantly reduced late leaf spot disease incidence and 
Francis et al. / Intl J Agric Biol, Vol 26, No 2, 2021

Table 5: Phytochemical compounds with antifungal activity obtained from ethyl acetate leaf extract of $J$. curcas

\begin{tabular}{|c|c|c|c|c|}
\hline Retention time (min) & Compound name & Molecular formula & Molecular weight (g/mol) & References \\
\hline 7.539 & 1,2,3-Ropanetriol, monoacetate & $\mathrm{C}_{5} \mathrm{H}_{10} \mathrm{O}_{4}$ & 134.13 & (Teoh and Mashitah 2012) \\
\hline 8.460 & 2,5-Pyrrolidinedione & $\mathrm{C}_{8} \mathrm{H}_{13} \mathrm{NO}_{2}$ & 331.32 & (Takayama et al. 1982) \\
\hline 8.826 & Hexadecane & $\mathrm{C}_{16} \mathrm{H}_{34}$ & 226.44 & (Adeleye et al. 2010) \\
\hline 9.273 & Methyl salicylate & $\mathrm{C}_{8} \mathrm{H}_{8} \mathrm{O}_{3}$ & 152.15 & (Pawar and Thaker 2006) \\
\hline 11.321 & Triacetin & $\mathrm{C}_{9} \mathrm{H}_{14} \mathrm{O}_{6}$ & 218.21 & (Osuntokun and Olajubu 2014) \\
\hline 11.813 & Heptadecane & $\mathrm{C}_{17} \mathrm{H}_{36}$ & 240.5 & (Zhang et al., 2015) \\
\hline 11.899 & 8-Hexadecenal, 14-methyl-, (Z)- & $\mathrm{C}_{17} \mathrm{H}_{32} \mathrm{O}$ & 252.4 & (Osuntokun and Olajubu 2014) \\
\hline 13.467 & Phenol,2,4-bis(1,1-dimethylethyl) & $\mathrm{C}_{17} \mathrm{H}_{30} \mathrm{OSi}$ & 278.50 & (Jun et al. 2018) \\
\hline 13.993 & 1-Naphthalenol & $\mathrm{C}_{10} \mathrm{H}_{8} \mathrm{O}$ & 144.17 & (Kumar et al. 2012) \\
\hline 14.503 & Hexadecane & $\mathrm{C}_{16} \mathrm{H}_{34}$ & 226.41 & (Oliveira et al. 2014) \\
\hline 15.658 & Heptadecane & $\mathrm{C}_{17} \mathrm{H}_{36}$ & 240.48 & (Zhang et al. 2015) \\
\hline 16.889 & E-14-Hexadecenal & $\mathrm{C}_{16} \mathrm{H}_{30} \mathrm{O}$ & 238.41 & (Devakumar et al. 2017) \\
\hline 17.106 & 1-Tetradecene & $\mathrm{C}_{14} \mathrm{H}_{28}$ & 196.37 & (Tayung and Jha 2014) \\
\hline 17.896 & Tetramethyl-2-hexadecen-1-ol & $\mathrm{C}_{20} \mathrm{H}_{40} \mathrm{O}$ & 296.50 & (Mohy and Mohyeldin 2018) \\
\hline 18.868 & $n$-Hexadecanoic acid & $\mathrm{C}_{16} \mathrm{H}_{32} \mathrm{O}_{2}$ & 256.42 & (Tyagi and Agarwal 2017) \\
\hline 19.109 & 5-Eicosene, $(\mathrm{E})$ - & $\mathrm{C}_{20} \mathrm{H}_{40}$ & 280.50 & (Adibe et al. 2019) \\
\hline 19.172 & Hexadecanoic acid ethyl ester & $\mathrm{C}_{18} \mathrm{H}_{36} \mathrm{O}_{2}$ & 284.47 & (Mohy and Mohyeldin 2018) \\
\hline 19.338 & 2-Methyl-Z,Z-3,13-octadecadienol & $\mathrm{C}_{19} \mathrm{H}_{36} \mathrm{O}$ & 280.50 & (Adibe et al. 2019) \\
\hline 20.179 & 9,17-Octadecadienal, (Z)- & $\mathrm{C}_{18} \mathrm{H}_{32} \mathrm{O}$ & 264.40 & (Adibe et al. 2019) \\
\hline 20.413 & Phytol & $\mathrm{C}_{20} \mathrm{H}_{40} \mathrm{O}$ & 296.54 & (Pejin et al. 2014) \\
\hline 21.008 & 9,12,15-Octadecatrienoic acid ethyl ester, (Z,Z,Z)- & $\mathrm{C}_{20} \mathrm{H}_{34} \mathrm{O}_{2}$ & 306.48 & (Mohy and Mohyeldin 2018) \\
\hline 21.186 & Heptadecanoic acid ethyl ester & $\mathrm{C}_{19} \mathrm{H}_{38} \mathrm{O}_{2}$ & 298.50 & (Bashir et al. 2019) \\
\hline 23.869 & Eicosane & $\mathrm{C}_{20} \mathrm{H}_{42}$ & 282.50 & (El-Naggar et al. 2017) \\
\hline
\end{tabular}

severity as compared to the lowest concentration. This finding corresponds with the finding of an investigation by Amah and Aliero (2009) who revealed that disease incidence and severity were reported as being low in plants treated with plant extract at the highest concentration.

Growth parameters varied significantly with the effect of treatments, solvents and concentration. The plants treated with Chlorothalonil and J. curcas had taller shoots and bigger number of leaves per plant at both flowering and maturity compared with the control. In addition, the plants treated with methanolic and chloroform extracts had taller shoots and bigger number of leaves per plant, at both flowering and maturity compared with ethyl acetate extracts. Moreover, yield attributes components varied significantly with $J$. curcas leaf extracts concentrations, where $J$. curcas leaf extracts at the highest concentration influenced groundnut yield similar to the standard fungicides (Chlorothalonil). This observation is consistent with the results by Ghewande (1989) who found leaf extracts of Azadirachta indica and Lawsonia inermis effective in managing both groundnut late leaf spot and rust diseases and increased yield by 15 $40 \%$ under field conditions. For this case, methanolic, ethyl acetate and chloroform leaf extracts of J. curcas were found effective against late leaf spot disease LLS subsequently improved the growth and yield of groundnuts compared with the control treatments.

GC-MS analysis was performed on chloroform, ethyl acetate and methanolic extracts of $J$. curcas since they exhibited the antifungal activity under in vivo experiment. The GC-MS identified the presence of different phytoconstituents from chloroform), ethyl acetate and methanolic leaf extracts of J. curcas. The qualitative differences of phytochemical constituents observed in this study may be attributed by different solvents employed for extraction. This observation corresponds with the findings by Kordali et al. (2009), who reported that, the spectra solubility of phytochemicals depends on the type of solvent used for extraction. In addition, phytochemical differences could be the result of the habitat for plant growth. This is consistent with Farooq et al. (2007) finding that, the phytochemical compounds composition depends on the plant habitat. The phytochemical analysis revealed the existence of octadecanoic acid; hexadecanoic acid methyl ester (palmitic acid); 9, 12-octadecadienoic acid (Z,Z) methyl ester and phytol in $J$. curcas leaf extracts. Amongst them hexadecanoic acid; octadecanoic acid methyl ester, and 9, 12-octadecadienoic acid (Z, Z) methyl ester are fatty acids, with the exception of phytol which is diterpene alcohol (Hema et al. 2011; Banaras et al. 2017). According to studies (Belakhdar et al. 2015; Chukwunonye et al. 2015), fatty acids possess antifungal property against diverse mycological pathogens. Since the fungal tissue is lipophilic in nature the fatty acids will attract the absorption of the fungus more easily (Inouye et al. 1999). Moreover, even the minor phytochemical components possibly contributed to antifungal effect by working synergistically with major compounds as reported by (Marino et al. 2001). The possession of these important phyto-compounds with antifungal properties in 
Management of Groundnut Late Leaf Spot Disease / Intl J Agric Biol, Vol 26, No 2, 2021

Table 6: Phytochemical compounds with antifungal activity obtained from methanolic leaf extract of J.curcas

\begin{tabular}{|c|c|c|c|c|}
\hline Retention time (min) & Compound name & Molecular formula & Molecular weight $(\mathrm{g} / \mathrm{mol})$ & References \\
\hline 7.539 & 1,2,3-Propanetriol monoacetate & $\mathrm{C}_{5} \mathrm{H}_{10} \mathrm{O}_{4}$ & 134.13 & (Teoh and Mashitah 2012) \\
\hline 9.273 & Methyl salicylate & $\mathrm{C}_{8} \mathrm{H}_{8} \mathrm{O}_{3}$ & 152.15 & (Essien et al. 2015) \\
\hline 10.549 & 2-Undecanone & $\mathrm{C}_{11} \mathrm{H}_{22} \mathrm{O}$ & 170.29 & (Bisht and Chanotiya 2011) \\
\hline 10.841 & Indole & $\mathrm{C}_{8} \mathrm{H}_{7} \mathrm{~N}$ & 117.15 & (Sumiya et al. 2017) \\
\hline 10.898 & Decanoic acid methyl ester & $\mathrm{C}_{11} \mathrm{H}_{22} \mathrm{O}_{2}$ & 186.29 & (Belakhdar et al. 2015) \\
\hline 11.121 & 2-Methoxy-4-vinylphenol & $\mathrm{C}_{9} \mathrm{H}_{10} \mathrm{O}_{2}$ & 150.17 & (Guo et al. 2008) \\
\hline 11.287 & Tert-hexadecanethiol & $\mathrm{C}_{16} \mathrm{H}_{34} \mathrm{~S}$ & 258.50 & (Yang et al. 2016) \\
\hline 11.653 & Phenol, 2,6-dimethoxy- & $\mathrm{C}_{8} \mathrm{H}_{10} \mathrm{O}_{3}$ & 154.16 & (Yang et al. 2016) \\
\hline 11.813 & Tetradecane & $\mathrm{C}_{14} \mathrm{H}_{30}$ & 198.39 & (Begum et al. 2016) \\
\hline 11.905 & Cyclotetradecane & $\mathrm{C}_{14} \mathrm{H}_{28}$ & 196.37 & (Afrouzan et al. 2018) \\
\hline 11.991 & Pentanoic acid ethyl ester & $\mathrm{C}_{7} \mathrm{H}_{14} \mathrm{O}_{2}$ & 130.18 & (Sumiya et al. 2017) \\
\hline 12.248 & 2-Propenoic acid 3-phenyl-, methyl ester & $\mathrm{C}_{10} \mathrm{H}_{10} \mathrm{O}_{2}$ & 162.18 & (Umaiyambigai et al. 2017) \\
\hline 12.334 & Diphenyl ether & $\mathrm{C}_{12} \mathrm{H}_{10}$ & 170.21 & (Zhang et al. 2018) \\
\hline 13.198 & Pentadecane & $\mathrm{C}_{15} \mathrm{H}_{32}$ & 212.41 & (Zhang et al. 2015) \\
\hline 13.272 & Tridecane & $\mathrm{C}_{13} \mathrm{H}_{28}$ & 184.36 & (Yuan et al. 2012) \\
\hline 14.503 & Hexadecane & $\mathrm{C}_{16} \mathrm{H}_{34}$ & 226.44 & (Oliveira et al. 2014) \\
\hline 16.706 & Heptadecane & $\mathrm{C}_{17} \mathrm{H}_{36}$ & 240.47 & (Musa et al. 2015) \\
\hline 16.797 & 17-Pentatriacontene & $\mathrm{C}_{35} \mathrm{H}_{70}$ & 490.93 & (Zhang et al. 2015) \\
\hline 16.889 & 1-Nonadecene & $\mathrm{C}_{19} \mathrm{H}_{38}$ & 266.50 & (Asong et al. 2019) \\
\hline 17.015 & E-15-Heptadecenal & $\mathrm{C}_{17} \mathrm{H}_{32} \mathrm{O}$ & 252.43 & (Begum et al. 2016) \\
\hline 17.192 & 8-Hexadecenal 14-methyl-, & $\mathrm{C}_{17} \mathrm{H}_{32} \mathrm{O}$ & 252.40 & (Aja et al. 2014) \\
\hline 17.787 & Cyclopentadecane & $\mathrm{C}_{15} \mathrm{H}_{30} \mathrm{O}$ & 210.40 & (Nakashima et al. 2014) \\
\hline 18.474 & Hexadecanoic acid methyl ester & $\mathrm{C}_{17} \mathrm{H}_{34} \mathrm{O}_{2}$ & 270.45 & (Belakhdar et al. 2015) \\
\hline 18.777 & 1-Octadecene & $\mathrm{C}_{18} \mathrm{H}_{36}$ & 252.48 & (Omoruyi et al. 2014) \\
\hline 18.868 & 2-Methyl-Z, Z-3, 13-octadecadienol & $\mathrm{C}_{19} \mathrm{H}_{36} \mathrm{O}$ & 280.49 & $\begin{array}{l}\text { (Phatangare } \text { et al. 2017; Adibe } \text { et al. } \\
\text { 2019) }\end{array}$ \\
\hline 18.983 & Oleic acid & $\mathrm{C}_{18} \mathrm{H}_{34} \mathrm{O}_{2}$ & 282.46 & (Walters et al. 2004) \\
\hline 19.486 & 9,17-Octadecadienal, (Z)- & $\mathrm{C}_{18} \mathrm{H}_{32} \mathrm{O}$ & 264.40 & (Adibe et al. 2019) \\
\hline 19.836 & 2-Methyl-Z,Z-3,13-octadecadienol & $\mathrm{C}_{19} \mathrm{H}_{36} \mathrm{O}$ & 280.28 & (Adibe et al. 2019) \\
\hline 20.288 & 9, 12-Octadecadienoic acid (Z,Z)-methyl ester & $\mathrm{C}_{19} \mathrm{H}_{34} \mathrm{O}_{2}$ & 294.47 & (Chukwunonye et al. 2015) \\
\hline 20.413 & Phytol & $\mathrm{C}_{20} \mathrm{H}_{40} \mathrm{O}$ & 296.0 & (Hema et al. 2011) \\
\hline 20.556 & Octadecanoic acid methyl ester & $\mathrm{C}_{19} \mathrm{H}_{38} \mathrm{O}_{2}$ & 298.50 & (Banaras et al. 2017) \\
\hline 21.129 & Behenic alcohol & $\mathrm{C}_{22} \mathrm{H}_{46} \mathrm{O}$ & 326.60 & (Chandrasekaran et al. 2011) \\
\hline 21.186 & Octadecanoic acid ethyl ester & $\mathrm{C}_{20} \mathrm{H}_{40} \mathrm{O}_{2}$ & 312.53 & (Mohy and Mohyeldin 2018) \\
\hline 21.380 & 3,7,11,15-Tetramethyl-2-hexadecen-1-ol & $\mathrm{C}_{20} \mathrm{H}_{40} \mathrm{O}$ & 296.53 & (Mohy and Mohyeldin 2018) \\
\hline 22.096 & 9,17-Octadecadienal, (Z)- & $\mathrm{C}_{18} \mathrm{H}_{32} \mathrm{O}$ & 264.40 & (Chukwunonye et al. 2015) \\
\hline 23.875 & Eicosane & $\mathrm{CH}$ & 282.50 & (Shirani et al. 2017) \\
\hline 24.241 & Docosanoic acid methyl ester & $\mathrm{C}_{23} \mathrm{H}_{46} \mathrm{O}_{2}$ & 354.61 & (Aida et al. 2016) \\
\hline
\end{tabular}

$J$. curcas leaf extracts signifies they are effective against fungal pathogens including $P$. personata.

\section{Conclusion}

The study concludes that the methanolic, ethyl acetate and chloroform leaf extracts of $J$. curcas contain important antifungal phytoconstituents such as hexadecane; $n$ hexadecanoic acid; phenol, 2,4 bis (-dimethylethyl); phytol and hexadecanoic methyl ester, which are responsible for the control of late leaf spot disease. Hence, methanolic, ethyl acetate and chloroform leaf extracts of $J$. curcas can be used as substitute biopesticides for inhibiting late leaf spot disease on groundnut.

\section{Acknowledgements}

We acknowledge DAAD (German Academic Exchange) and CREATES, Tanzania for their financial support.

\section{Author Contributions}

MF developed and planned the study, MF, EM and MC statistically analysed the data MF, PN and EM, interpreted the results and MF made write up.

\section{Conflict of Interest}

Authors declared no conflicts of interest.

\section{Data Availability}

The research data can be obtained through concerting the corresponding author.

\section{Ethics Approval}

The ethical approval was obtained from the Tropical Pesticide Research Institute under Herbarium section, Arusha. 


\section{References}

Adeleye IA, FV Daniels, M Omadime (2010). Characterization of volatile components of epa-ijebu: A native wonder cure recipe. $J$ Pharmacol Toxicol 6:97-100

Adibe MK, MG IbokAdeniyi-Akee, A Mukaram, OE Ajala (2019). Chemical compositions and antioxidant activity of leaf and stem essential oils of Bryophyllum pinnatum (Lam.) Kurz. GSC Biol Pharm Sci 9:57-64

Afrouzan HT, Z Azar, Z Sedigheh, E Ali (2018). Chemical composition and antimicrobial activities of Iranian propolis. Iran Biomed J 22:50-65

Aida HS, AA Safaa, MB Khouloud (2016). GC-MS spectroscopic approach and antifungal potential of bioactive extracts produced by marine macro algae. Egypt J Aquat Res 42:289-299

Aja PM, N Nwachukwu, UA Ibiam, IO Igwenyi, CE Offor, UO Orji (2014). Comparative gas chromatography-mass spectrometry (GCMS) analysis of chemical compounds of Moringa oleifera leaves and Seeds from Abakaliki, Nigeria. Adv Life Sci Technol 24:73-79

Amah CP, AA Aliero (2009). Efficacy of aqueous extracts of some selected medicinal plants in the control of Fusarium oxysporum. Biotr Res Intl J 1:39-43

Ashraf SA, E Al-Shammari, T Hussain, S Tajuddin, BP Panda (2017). In-vitro antimicrobial activity and identification of bioactive components using GC-MS of commercially available essential oils in Saudi Arabia. J Food Sci Technol 54:3948-3958

Asong JA, PT Ndhlovu, NS Khosana, AO Aremu, W Otang-Mbeng (2019). Medicinal plants used for skin-related diseases among the Batswanas in Ngaka Modiri Molema District Municipality, South Africa. South Afr J Bot 126:11-20

Banaras S, A Javaid, IH Khan (2021). Bioassays guided fractionation of Ageratum conyzoides for identification of natural antifungal compounds against Macrophomina phaseolina. Intl J Agric Biol 25:761-767

Banaras S, A Javaid, A Shoaib, E Ahmed (2017). Antifungal Activity of Cirsium arvenseextracts against phytopathogenic fungus Macrophomina phaseolina. Planta Danin 35; Article e017162738

Bashir S, K Jabeen, KS Iqbal, S Javed, A Naeem (2019). Lantana camara: Phytochemical analysis and antifungal prospective. Planta Danin 37; Article e019193526

Begum FI, R Mohankumar, M Jeevan, K Ramani (2016). GC-MS analysis of bio-active molecules derived from Paracoccus pantotrophus FMR19 and the antimicrobial activity against bacterial pathogens and MDROs. Ind J Microbiol 56:426-432

Belakhdar G, AE Benjouad, H Abdennebi (2015). Determination of some bioactive chemical constituents from Thesium humile Vahl $J$ Mat Environ Sci 6:2778-2783

Bisht D, CS Chanotiya (2011). 2-Undecanone rich leaf essential oil from Zanthoxylum armatum. Nat Prod Commun 6:111-114

Bouzabata A, F Mahomoodally, C Tuberoso (2018). Ethnopharmacognosy of Echinops spinosus L. in North Africa: A mini review. J Compl Med Res 8:40-52

Bughio SH, QS Muhammad, MShahabuddin, B Shaista, AM Moina, AM Ayaz (2017). Chemical composition of the essential oils from Tamarix dioica and determination of its antibacterial activity. Intl J Food Propert 20:2660-2667

Campa C, D Kuhn, D Diouf, C Valentin, R Manlay (2008). Taxonomy And Biology of the Tropical Plant Jatropha curcas L., pp:1-15. Vanatrop Workshop, Montpellier, France

Chandrasekaran M, A Senthilkumar, V Venkatesalu (2011). Antibacterial and antifungal efficacy of fatty acid methyl esters from the leaves of Sesuvium portulacastrum L. Eur Rev Med Pharmacol Sci 15:775-780

Chiteka ZA, TA Kucharek, DA Knauft, DW Gorbet, FM Shokes (1988). Components of resistance to late leafspot in peanut. I. Levels and variability implications for selection. Peanut Sci 15:25-30

Chukwunonye MO, IN Kelechi, NE Marycolette (2015). The chemical constituents and Bioactivity of the seed (Fruit) extracts of Buchholzia coriacea Engler (Capparaceae). J Appl Sci Environ Manage 19:795-801
Dada EO, FO Ekundayo, OO Makanjuola (2014). Antibacterial activities of Jatropha curcas (Linn) on coliforms isolated from surface waters in Akure, Nigeria. Intl J Biomed Sci 10:25-30

Devakumar J, V Keerthan, SS Sudha (2017). Identification of bioactive compounds by gas chromatography-mass spectrometry analysis of Syzygium jambos L. collected from Western Ghats region Coimbatore, Tamilnadu. Asian J Pharm Clin Res 10:364-369

Ebrahimabadi AH, MM Movahedpour, H Batooli, EH Ebrahimabadi, A Mazoochi, MM Qamsari (2016). Volatile compounds analysis and antioxidant, antimicrobial and cytotoxic activities of Mindium laevigatum. Iran J Basic Med Sci 19:1337-1344

El-Naggar NEA, AAA El-Bindary, M Abdel-Mogib , NS Sour (2017). In vitro activity, extraction, separation and structure elucidation of antibiotic produced by Streptomyces anulatus NEAE-94 active against multidrug-resistant Staphylococcus aureus. Biotechnol Biotechnol Equip 31:418-430

Engindeniz S, GC Ozturk (2013). An economic comparison of pesticide application for processing and table tomatoes, a case study for Turkey. J Plant Prot Res 53:230-237

Essien E, JS Newby, TM Walker, WN Setzer, O Ekundayo (2015). Characterization and antimicrobial activity of volatile constituents from fresh fruits of Alchornea cordifolia and Canthium subcordatum. Medicines 3; Article 1

Farooq A, TA Sajid, LA Muhammad, HG Anwarul (2007). Moringa oleifera: A food plant with multiple medicinal uses. Phytother Res 21:17-25

Garcia RP, P Lawas (1990). Potential plant extracts for the control of Azolla fungal pathogens. Phil Agric 73:343-348

Ghewande MP (1989). Management of foliar diseases of groundnut (Arachis hypogaea) using plant extracts. Ind J Agric Sci 59:133134

Guo L, W Jin-zhong, H Ting, C Tong, R Khalid, Q Lu-ping (2008). Chemical composition, antifungal and antitumor properties of ether extracts of Scapania verrucosa Heeg and its endophytic fungus Chaetomium fusiforme. Molecules 13:2114-2125

Hema R, S Kumaravel, K Alagusundaram (2011). GC/MS Determination of Bioactive components of Murraya koenigii. $J$ Amer Sci 7:80-83

Igbinosa OO, EO Igbinosa, OA Aiyegoro (2009). Antimicrobial activity and phytochemical screening of stem bark extracts from Jatropha curcas L. Afr J Pharm Pharmacol 3:58-62

Inouye S, K Uchida, H Yamaguchi (1999). In-vitro and in-vivo anti trichophyton activity of essential oils by vapour contact. Mycoses 44:99-107

Javaid A, SF Naqvi, IH Khan (2021). Ethyl acetate extract of Chenopodium murale root, a source of bioactive compounds. Pak $J$ Weed Sci Res 27:93-100

Javaid A, R Munir, IH Khan, A Shoaib (2020). Control of the chickpea blight, Ascochyta rabiei, with the weed plant, Withania somnifera. Egypt J Biol Pest Cont 30; Article 114

Jordan DL, RL Brandenburg, AB Brown, GS Bullen, GT Roberson, B Shew, FJ Spears (2012). Peanut Information, pp:100-127. North Carolina Cooperative Extension Service, College of Agriculture \& Life Sciences, North Carolina State University, Raleigh, North Carolina, USA

Jun M, X Rui-Rui, L Ya, R Di-Feng, L Jun (2018). Composition, antimicrobial and antioxidant activity of supercritical fluid extract of Elsholtzia ciliate. J Essent Oil Bear Plants 21:556-562

Kalimuthu K, S Vijayakumar, R Senthilkumar (2010). Antimicrobial activity of the Biodiesel Plant, Jatropha curcas L. Intl J Pharm Biosci 3:1-5

Kayondo SI, PR Rubaihayo, BR Ntare, PT Gibson, Edema, RA Ozimati, DK Okello (2014). Genetics of resistance to groundnut rosette virus disease. Afr Crop Sci J 22:21-30

Khan IH, A Javaid (2020). Anticancer, antimicrobial and antioxidant compounds of quinoa inflorescence. Adv Life Sci 8:68-72

Khan IH, A Javaid, AH Al-Taie, D Ahmed (2020). Use of neem leaves as soil amendment for the control of collar rot disease of chickpea. Egypt J Biol Pest Cont 30; Article 98 
Khedikar YP, MVC Gowda, C Sarvamangala, KV Patgar, HD Upadhyaya, RK Varshney (2010). A QTL study on late leaf spot and rust revealed one major QTL for molecular breeding for rust resistance in groundnut (Arachis hypogaea L.). Theoret Appl Genet 121:971-984

Kishore GK, S Pande, S Harish (2007). Evaluation of essential oils and their components for broad-spectrum antifungal activity and control of late leaf spot and crown rot diseases in peanut. Plant Dis 91:375-379

Kordali S, A Cakir, TA Akcin, E Mete, A Akcin, T Aydin, H Kilic (2009). Antifungal and herbicidal properties of essential oils and $n$-hexane extracts of Achillea gypsicola Hub-Mor. And Achillea biebersteinii A fan. (Asteraceae). Ind Crops Prod 29:562-570

Kumar S, P Kumar, N Sati (2012). Synthesis and Biological evaluation of schiff bases and azetidinones of 1-naphtol. J Pharm Biopest 4:246-249

Manikandan G, RA Vimala, C Divya, R Ramasubbu (2017). GC-MS Analysis of phytochemical constituents in the petroleum ether leaf extracts of Millettia peguensis. Intl Res J Pharm 8:455-458

Marino M, C Bersani, G Comi (2001). Impedance measurements to study the antimicrobial activity of essential oils from Lamiaceae and Compositae. Intl J Food Microbiol 67:187-195

Mohy SMED, MM Mohyeldin (2018). Component analysis and antifungal activity of the compounds extracted from four brown seaweeds with different solvents at different seasons. J Ocean Univ Chin 17:1178-1188

Monfort WS, AK Culbreath, KL Stevenson, TB Brenneman, DW Gorbet, SC Phatak. (2004). Effects of reduced tillage, resistant cultivars, and reduced fungicide inputs on progress of early leaf spot of peanut (Arachis hypogaea). Plant Dis 88:858-864

Musa AM, MA Ibrahim, AB Aliyu, MS Abdullahi, N Tajuddeen, H Ibrahim, AO Oyewale (2015). Chemical composition and antimicrobial activity of hexane leaf extract of Anisopus mannii (Asclepiadaceae). J Intercult Ethnopharm 4:129-133

Naidu RA, FM Kimmins, CM Deom, P Subrahmanyam, AJ Chiyembekeza, PJAVD Merwe (1999). Groundnut rossette: A virus disease affecting groundnut production in sub-Saharan Africa. Plant Dis 83:700-709

Nakashima TN, I Masato, O Junya, K Yoshiyuki, N Kenichiro, M Atsuko, I Aki, O Kazuhiko, S Kazuro, T Yoko, O Satoshi (2014). Mangromicins A and B: Structure and antitrypanosomal activity of two new cyclopentadecane compounds from Lechevalieria aerocolonigenes K10-0216. J Antibiot 67:253-260

Nwosu MO, JI Okafor (1995). Preliminary studies of the antifungal activities of some medicinal plants against Basidiobolus and some other pathogenic fungi. Mycoses 38:191-195

Oliveira GT, MS Jaqueline, LHEP Rosa, S Siqueira, S Johann, LARS Lima (2014). In vitro antifungal activities of leaf extracts of Lippia alba (Verbenaceae) against clinically important yeast species. Rev Soc Bras Med Trop 47:247-250

Omoruyi BE, AJ Afolayan, G Bradley (2014). Chemical composition profiling and antifungal activity of the essential oil and plant extracts of Mesembryanthemum edule (L.) bolus leaves. Afr $J$ Trad Compl Altern Med 11:19-30

Osei K, JY Asibuo, A Agyeman, P Osei-Bonsu, Y Danso, J Adomako (2013). Reactions of some confectionery groundnut accessions to plant parasitic nematodes infection. Agrosearch 13:1-11

Osuntokun OT, FA Olajubu (2014). Comparative study of phytochemical and proximate analysis of seven Nigerian medicinal plants. Appl Sci Res J 2:10-26

Passos XS, CM An, SP Juliana, CF Ana, FC Garcia, RS Maria (2003). composition and antifungal activity of the essential oils of Caryocar brasiliensis. Pharm Biol 41:319-324

Pasupuleti J, SN Nigam, MK Pandey, P Nagesh, RK Varshney (2013). Groundnut improvement: Use of genetic and genomic tools. Front Plant Sci 4:23-38

Pawar VC, VS Thaker (2006). In vitro efficacy of oils against Aspergillus niger. Mycosis 49:316-323

Pejin BSA, M Sokovi, J Glamoclij, A Ciri, MM Nikoli (2014). Further in vitro evaluation of antiradical and antimicrobial activities of phytol. Nat Prod Res 28:372-376
Phatangare ND, KK Deshmukh, VD Murade, PH Naikvade, DP Hase, MJ Chavhan, HE Velis (2017). Isolation and characterization of phytol from Justicia gendarussa Burm. f.-An anti-Inflammatory compound. Intl J Pharm Phytochem Res 9:864-872

Philipo M, S Nchimbi-Msolla (2019). Effect of watering regimes on yield and agronomic traits of exotic groundnut genotypes in Tanzania. $J$ Adv Biol Biotechnol 21:1-6

Prasad L, S Pradhan, LM Das and SN Naik (2012). Experimental assessment of toxic phorbol ester in oil, biodiesel and seed cake of Jatropha curcas and use of biodiesel in diesel engine. Appl Ener 93:245-250

Ruban P, K Gajalakshmi (2012). In vitro antibacterial activity of Hibiscus rosa-sinensis flower extract against human pathogens. Asian Pac J Trop Biomed 2:399-403

Saeed I, MF Hassan (2009). High yielding groundnut (Arachis hypogea L.) variety Golden. Pak J Bot 41:2217-2222

Saetae D, S Worapot (2010). Antifungal activities of ethanolic extract from Jatropha curcas. seed cake. J Microbiol Biotechnol 20:319324

Sharma M, SS Suman, RD Agrawal (2019). Isolation and identification of phytosterols from Anogeissus pendula (Edgew) and their antimicrobial potency. J Pharmacogn Phytochem 8:1665-1670

Shirani M, A Samimi, H Kalantari, M Madani, ZA Kord (2017). Chemical composition and antifungal effect of hydroalcoholic extract of Allium tripedale against Candida species. Curr Med Mycol 3:6-12

Subrahmanyam P, D McDonald, F Waliyar, LJ Reddy, SN Nigam, RW Gibbons, PS Rao (1995). Screening methods and sources of resistance to rust and late leaf spot of groundnut. Information Bulletin no. 47. International Crops Research Institute for the Semi-Arid Tropics, Hyderabad, India

Sumiya T, I Mai, O Keimei (2017). Synthesis of imidazole and indole hybrid molecules and antifungal activity against rice blast. Intl $J$ Chem Eng Appl 8:233-236

Takayama C, F Akira, K Osamu, H Yoshio (1982). Quantitative structureactivity relationships of antifungal 1 - (3, 5-dichlorophenyl)-2, 5pyrrolidinediones and 3- (3, 5-Dichlorophenyl)-2, 4oxazolidinedionest. Agric Biol Chem 46:2755-2758

Tayung K, DK Jha (2014). Endophytic fungi as potential sources of bioactive natural products: Prospects and challenges. Jodhpur Rev Plant Pathol 6:299-334

Teoh PY, MD Mashitah (2012). Screening of antifungal activities from genera Trametes against growth of selected wood-degrading fungi from Malaysia. Aust J Basic Appl Sci 6:79-85

Thangavelu R, P Sundararaju, S Sathiamoorthy (2004). Management of anthracnose disease of banana caused by Colletotrichum musae using plant extracts. J Hortic Sci Biotechnol 79:664-668

Tsigbey FK, RL Brandenburg, VA Clottey (2003). Peanut production methods in Northern Ghana and some disease perspectives. World Geog Peanut Knowl Base Web 9:33-38

Tshilenge-Lukanda L, A Kalonji-Mbuyi, KKC Nkongolo, RV Kizungu (2013). Effect of gamma irradiation on morpho-agronomic characteristics of groundnut (Arachis hypogaea L.). Amer J Plant Sci 4:2186-2192

Tyagi T, M Agarwal (2017). GC-MS analysis of invasive aquatic weed, Pista Stratiotes L. and Eichhornia crassipes (Mart.) Solms. Intl J Curr Pharm Res 9:111-117

Umaiyambigai D, K Saravanakumar, RG Adaikala (2017). Phytochemical profile and antifungal activity of leaves methanol extract from the Psydrax dicoccos (Gaertn) Teys. \& Binn. Rubiaceae family. Intl J Pharm Phytochem Ethnomed 7:53-61

Vijayabaskar G, V Elango (2018). Determination of phytocompounds in Withania somnifera and Smilax china using GC-MS technique. $J$ Pharm Phytochem 7:554-557

Walters D, L Raynor, A Mitchell (2004). Antifungal activities of four fatty acids against plant pathogenic fungi. Mycopathology 157:87-90

Wanxi P, L Zhi, C Junbo, G Fangliang, Z Xiangwei (2013). Biomedical molecular characteristics of YBSJ extractives from Illicium verum fruit. Biotechnol Biotechnol Equip 27:4311-4316 
Francis et al. / Intl J Agric Biol, Vol 26, No 2, 2021

Yang J, Y Cheng-Hong, L Ming-Tsai, G Zi-Jie, W Yuh-Wern, C Li-Yeh (2016). Chemical composition, antioxidant, and antibacterial activity of wood vinegar from Litchi chinensis. Molecules 21:1150-1159

Yuan J, W Raza, Q Shen, Q Huang (2012). Antifungal activity of Bacillus amyloliquefaciens NJN-6 volatile compounds against Fusarium oxysporum f. spp. cubense. Appl Environ Microbiol 78:5942-5944
Zhang P, X Li, XL Yuan, YM Du, BG Wang, ZF Zhang (2018). Antifungal prenylated diphenyl ethers from Arthrinium arundinis and endophytic fungus isolated from the leaves of tobacco (Nicotiana tabacum L.). Molecules 23:3179-3185

Zhang XX, C Xia, CJ Li, ZB Nan (2015). Chemical composition and antifungal activity of the volatile oil from Epichloë gansuensis, endophyte-infected and non-infected Achnatherum inebrians. Sci Chin Life Sci 58:512-514 